\title{
Critical Quantum Metrology with a Finite-Component Quantum Phase Transition
}

\author{
Louis Garbe, ${ }^{1}$ Matteo Bina $\odot,{ }^{2}$ Arne Keller, ${ }^{1,3}$ Matteo G. A. Paris $\odot,{ }^{2}$ and Simone Felicetti $\odot$, ${ }^{*}$ \\ ${ }^{1}$ Université de Paris, Laboratoire Matériaux et Phénomènes Quantiques UMR 7162, CNRS, 75013, Paris, France \\ ${ }^{2}$ Quantum Technology Lab, Dipartimento di Fisica Aldo Pontremoli, Università degli Studi di Milano, I-20133 Milano, Italy \\ ${ }^{3}$ Université Paris-Saclay, 91405 Orsay, France \\ ${ }^{4}$ Departamento de Física Teórica de la Materia Condensada and Condensed Matter Physics Center (IFIMAC), \\ Universidad Autónoma de Madrid, E-28049 Madrid, Spain
}

(Received 15 November 2019; accepted 5 March 2020; published 27 March 2020)

\begin{abstract}
Physical systems close to a quantum phase transition exhibit a divergent susceptibility, suggesting that an arbitrarily high precision may be achieved by exploiting quantum critical systems as probes to estimate a physical parameter. However, such an improvement in sensitivity is counterbalanced by the closing of the energy gap, which implies a critical slowing down and an inevitable growth of the protocol duration. Here, we design different metrological protocols that exploit the superradiant phase transition of the quantum Rabi model, a finite-component system composed of a single two-level atom interacting with a single bosonic mode. We show that, in spite of the critical slowing down, critical quantum optical probes can achieve a quantum-enhanced time scaling of the sensitivity in frequency-estimation protocols.
\end{abstract}

DOI: 10.1103/PhysRevLett.124.120504

In a system close to a critical point, small variations of physical parameters may lead to dramatic changes in the equilibrium state properties. The possibility of exploiting this sensitivity for metrological purposes is well known, and it has already been applied in classical devices, e.g., in superconducting transition-edge sensors [1]. Besides, the development of quantum metrology has extensively shown that quantum states can outperform their classical counterparts for sensing tasks [2]. Therefore, a question naturally arises: what sensitivity can be achieved using interacting systems close to a quantum-critical point?

In the last few years, this question has attracted growing interest and it has been addressed by different methods [3-11]. Many of these studies may be roughly divided in two approaches. One of them is based on the time evolution induced by a Hamiltonian close to a critical point $[3,4]$. In this approach, one prepares a probe system in a suitably chosen state, lets it evolve following its critical Hamiltonian, and finally measures it. This bears close similarity to the standard interferometric paradigm of quantum metrology [2]. The other approach is based on equilibrium properties of critical systems [5-7,12]. It consists of preparing and measuring the system ground state in the Hamiltonian case, or the system steadystate when driven-dissipative systems are considered. In proximity of the phase transition the susceptibility of the equilibrium state diverges, suggesting that an arbitrarily large estimation precision could be achieved. Unfortunately, the time required to prepare the equilibrium state also diverges, both in the Hamiltonian [13] and in the driven-dissipative case [14,15], a behavior called critical slowing down.
Recent results suggest that the dynamical and equilibrium approaches are equivalent for a large class of spin systems [9]. It was shown that both approaches make it possible to achieve the optimal scaling limit of precision with respect to system size and protocol duration. These results were obtained considering spin systems that undergo quantum phase transitions in the thermodynamic limit, where the number of constituents goes to infinity. Another interesting class of quantum critical systems is provided by light-matter interaction models [16], for which superradiant quantum phase transitions can be controllably implemented $[17,18]$. Recently, it has been theoretically shown that quantum phase transitions can appear also in quantum-optical systems with only a finite number of components [19-22], where the thermodynamic limit can be replaced by a scaling of the system parameters [23].

In this Letter, we assess the metrological potential of a finite-component quantum phase transition, explicitly taking into account the protocol duration. We design parameter-estimation protocols based on equilibrium properties, considering both the Hamiltonian and the dissipative setting. In particular, we consider the quantum Rabi model [21], which exhibits a superradiant phase transition despite involving only one spin interacting with a single bosonic field. We find analytical expressions for the scaling of the quantum Fisher information, and we find that this approach allows one to measure both spin and bosonic frequency with a favorable time scaling, in spite of the critical slowing down. In particular, we show that for spin frequency estimation our protocol exhibits time-scaling enhancement with respect to the paradigmatic Ramsey protocol [24,25], 
while for bosonic frequency estimation it saturates the Heisenberg limit.

Hamiltonian case.-Let us consider a spin interacting with a single bosonic mode according to the quantum Rabi Hamiltonian:

$$
\hat{H}=\omega_{0} \hat{a}^{\dagger} \hat{a}+\Omega \hat{\sigma}_{z}+\lambda\left(\hat{a}^{\dagger}+\hat{a}\right) \hat{\sigma}_{x},
$$

where $\omega_{0}$ is the frequency of the bosonic field, $\hat{a}$ and $\hat{a}^{\dagger}$ are creation and annihilation operators of the field, $\hat{\sigma}_{x}$ and $\hat{\sigma}_{z}$ are Pauli matrices associated with the spin, and $\lambda$ is the coupling parameter. We also define the renormalized coupling parameter $g \equiv \lambda / \sqrt{\Omega \omega_{0}}$. In the limit $\eta \equiv \omega_{0} / \Omega \rightarrow 0$, this system exhibits a phase transition at $g=1[21,22]$. We will analyze different critical quantum-metrology protocols that exploit this phase transition to estimate either the $\operatorname{spin}(\Omega)$ or the field $\left(\omega_{0}\right)$ frequency, assuming in each case that all other parameters are known. In particular, we consider the following three-step protocol: first, the system is initialized in its ground state for $g=0$; then, an adiabatic sweep is performed varying the parameter $g$ from 0 to some desired value close to the critical point $g=1$; finally, the measurement of a relevant observable is performed. The measurement results can then be used to estimate the desired physical parameter, as sketched in Fig. 1(a).

In order to evaluate the performances of these protocols, we need first to characterize the system ground state as a function of the system parameters. In the limit $\eta \rightarrow 0$, the system can be diagonalized using a Schrieffer-Wolff transformation [21]. We apply the unitary $\hat{U}=e^{i g \sqrt{\eta}\left(\hat{a}^{\dagger}+\hat{a}\right) \hat{\sigma}_{y}}$ to Eq. (1), which gives $\hat{H}_{N}=\hat{U} \hat{H} \hat{U}^{\dagger}$, where

$$
\hat{H}_{N}=\omega_{0} \hat{a}^{\dagger} \hat{a}+\Omega \hat{\sigma}_{z}+\frac{\omega_{0}}{2} g^{2} \hat{\sigma}_{z}\left(\hat{a}+\hat{a}^{\dagger}\right)^{2},
$$

(a)
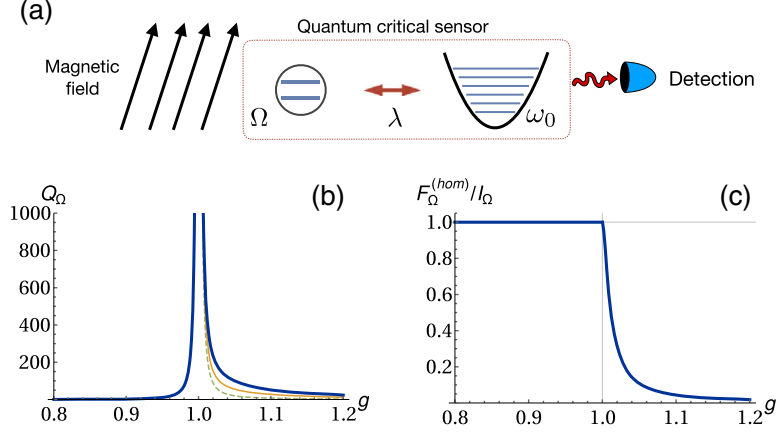

FIG. 1. (a) Schematic representation of the parameter-estimation protocol, where the quantum critical sensor is used to estimate the intensity of an external magnetic field. (b) Signalto-noise ratio $Q_{\Omega}$ versus $g$ for the estimation of $\Omega$, setting $\eta=0.1$ (thin dashed line), 0.2 (thin full line), and 0.01 (thick line). In the normal phase, $Q_{\Omega}$ is independent of $\eta$ for the value considered. In the superradiant phase, there is a small correction which depends on $\eta$, and that becomes negligible near the critical point. (c) Ratio FI/QFI for homodyne measurement of the $\hat{p}$ quadrature. In the normal phase $g<1$, the Cramer-Rao bound is attained for all values of $g$. up to terms $O\left(\omega_{0} \sqrt{\eta}\right)$. The effective Hamiltonian $\hat{H}_{N}$ provides a faithful description of the system ground state in the normal phase of the model. It is stable for $g<1$, whereas for $g \rightarrow 1$ the system experiences a phase transition towards the superradiant phase. Here, we will focus on the normal phase only, however, equivalent results can be found applying the same methods to the superradiant phase (see Supplemental Material [26]). In the normal phase, we can diagonalize $\hat{H}_{N}$ by projection in the lower spin eigenspace and Bogoliubov transformation. The ground state is given by $\left|\psi_{N}\right\rangle\left(\lambda, \Omega, \omega_{0}\right)=\hat{S}(\xi)|0\rangle \otimes|\downarrow\rangle$ up to terms $O(\sqrt{\eta})$. We defined $\xi=-\frac{1}{4} \log \left(1-g^{2}\right)$, while $\hat{S}(\xi)=\exp \left\{(\xi / 2)\left(\hat{a}^{\dagger}\right)^{2}-\left(\xi^{*} / 2\right) \hat{a}^{2}\right\}$ is a squeezing operator. The squeezing parameter diverges at the critical point, whereas the spin fluctuations are negligible, due to the much larger spin frequency. In turn, the excitation energy $\epsilon_{N}=\omega_{0} \sqrt{1-g^{2}}$ vanishes at the transition.

We are interested in the precise estimation of $A$ (with $A=\Omega$ or $\omega_{0}$ ) obtained by performing measurements on the ground state of the system. This precision is bounded by the quantum Cramer-Rao (CR) bound: $\delta^{2} A \geq\left(\nu \mathcal{I}_{A}\right)^{-1}$, where $\nu$ is the number of independent measurement rounds, and $\mathcal{I}_{A}$ is the quantum fisher information (QFI) relative to the parameter of interest $A$. Since the system is in a pure state, the QFI may be computed exactly as $\mathcal{I}_{A}=$ $4\left[\left\langle\partial_{A} \psi_{N} \mid \partial_{A} \psi_{N}\right\rangle+\left(\left\langle\partial_{A} \psi_{N} \mid \psi_{N}\right\rangle\right)^{2}\right]$. Close to the quantum phase transition, the dominant term of the QFI is

$$
\mathcal{I}_{A} \sim \frac{1}{32 A^{2}(1-g)^{2}},
$$

which means that the estimation of $\omega_{0}$ and $\Omega$ will yield the same signal-to-noise ratio $Q_{A}=A^{2} \mathcal{I}_{A}$. Equation (3) shows that $\mathcal{I}_{A}$ diverges at the critical point $g=1$, i.e., an arbitrarily large estimation precision could, in principle, be obtained. This is consistent with previous studies on critical metrology in light-matter systems $[6,7,27,28]$. To verify whether this bound is saturable with standard observables, we also study the Fisher information (FI) of homodyne detection on the field only. This is illustrated in Fig. 1, where we show $Q_{\Omega}$ versus $g$ for different values of $\eta$ [panel (a)], and the ratio FI/QFI for homodyne detection of the $\hat{p}$ quadrature [panel (b)]. In the normal phase, this homodyne measurement saturates the Cramer-Rao bound for all values of $g$. The $\hat{x}$ quadrature is always optimal also in the superradiant phase and, in general, we found that most quadratures allow one to achieve significant fractions of the optimal precision in proximity of the critical point (see Supplemental Material [26]). Notice that the homodyne signature of the ground state will be a random signal centered around 0 . The relevant information is encoded in the variance of this signal. Therefore, instead of averaging out the random signal, we need to reconstruct its statistics. This task requires repeated measurements; as the number of 
measurements $\nu$ increases, so will the precision, as per the CR bound.

Analysis of resources.-Let us now assess the performances of the proposed critical protocol against standard quantum metrology protocols. For the estimation of the bosonic frequency $\omega_{0}$, a benchmark is provided by interferometric protocols involving a phase difference $\Delta \phi=\omega_{0} T$, where $T$ is the evolution time. To ensure a fair comparison, we must carefully account for the resources needed to implement the critical and interferometric protocols. The relevant quantities to be considered are the system evolution time $T$ and the average number of photons involved $\langle\hat{N}\rangle$. A lossless interferometric protocol has a precision limited by the Heisenberg limit $\mathcal{I}_{\omega_{0}} \sim\langle\hat{N}\rangle^{2} T^{2}$. For the proposed critical protocol, we can readily compute $\langle\hat{N}\rangle:\left\langle\psi_{N}|\hat{N}| \psi_{N}\right\rangle=\sinh \xi^{2} \sim \frac{1}{4}\left(1-g^{2}\right)^{-1 / 2}$. Regarding the duration $T$ of the protocol, the relevant contribution is given by the time required to perform the adiabatic evolution. Because of the closing energy gap, the time needed to perform the adiabatic protocol diverges as the critical point is approached. However, the protocol time duration can be optimized considering a general adaptative process during which $g$ evolves with a speed $v(g)=d g / d t$.

Using adiabatic evolution theory (see the Supplemental Material [26]), we look for optimal adiabatic procedures that minimize the evolution time while ensuring that the system will remain in the ground state during the evolution. We find the following condition on the speed $v$ of evolution $v(g) \sim \gamma \omega_{0}\left(1-g^{2}\right)^{3 / 2}$, where $\gamma<1$ is a parameter which controls the probability of exciting the system. As a result, the time needed to sweep the coupling constant from 0 to some value $g \sim 1$ is given by

$$
T=\int_{0}^{g} \frac{d s}{v(s)} \sim \gamma^{-1} \omega_{0}^{-1}(1-g)^{-1 / 2} .
$$

This expression indeed diverges when $g$ goes to 1 . Upon inserting the expressions for $\langle\hat{N}\rangle$ and $T$ into Eq. (3), we find

$$
\mathcal{I}_{\omega_{0}} \sim \gamma^{2}\langle\hat{N}\rangle^{2} T^{2}
$$

i.e., the critical protocol allows one to estimate $\omega_{0}$ with the same precision granted by interferometric protocols. In other words, in spite of the critical slowing down, our critical protocol in the Hamiltonian case achieves the optimal Heisenberg-scaling precision for continuous-variable systems, with respect to both energy and time. Similar results have so far been obtained only for spin systems in the thermodynamic limit [9]. Concerning the estimation of the spin frequency, a natural benchmark is given by Ramsey interferometry with a single spin. For noiseless Ramsey interferometry, QFI scales like $T^{2}$ [24,25]. By contrast, in the critical case, we found using Eqs. (3) and (4),

$$
\mathcal{I}_{\Omega} \sim \frac{\gamma^{4} \omega_{0}^{4}}{8 \Omega^{2}} T^{4}
$$

i.e., our protocol achieves quartic scaling in the duration of the protocol, while Ramsey interferometry only scales quadratically. This is an unambiguous demonstration of time-scaling enhancement for a critical metrological protocol in light-matter system. Note, however, that the prefactor in Eq. (6) is very small, meaning that the critical protocol could outperform Ramsey only for large time $T$, that is, when operating very close to the critical point.

Dissipative process.-The above results are valid for isolated systems. However, decoherence due to the interaction with the environment generally reduces the performances of metrological protocols. In order to assess our protocol in realistic conditions, let us now consider the presence of both photon loss and spin decay. The dissipative dynamics of the system is described by a master equation (ME) of the form $\partial_{t} \hat{\rho}=-i[\hat{H}, \hat{\rho}]+\kappa L[\hat{a}] \hat{\rho}+$ $\Gamma L\left[\hat{\sigma}_{-}\right] \hat{\rho}$, where the Lindblad terms read $L[\hat{A}] \hat{\rho}=2 \hat{A} \hat{\rho} \hat{A}^{\dagger}-$ $\left\{\hat{A}^{\dagger} \hat{A}, \hat{\rho}\right\}$. Notice that we are considering a phenomenological master equation as we are interested in effective implementations of the model [29]. In order to characterize the dissipative case, we will first generalize the results obtained in Ref. [30] to include spin decay; details can be found in the Supplemental Material [26]. We assume $\kappa / \omega_{0}=O(1)$ and $\Gamma / \Omega=O(1)$, however our results can be extended to smaller dissipation values. By considering the spin-decay term explicitely and using Schrieffer-Wolff transformation, we decouple the spin and field, and project the spin into the $|\downarrow\rangle\langle\downarrow|$ subspace. This yields an effective $\mathrm{ME}$ for the state of the bosonic field:

$$
\begin{aligned}
\partial_{t} \hat{\rho}_{b d}= & -i\left[\omega_{0} \hat{a}^{\dagger} \hat{a}-Y\left(\hat{a}+\hat{a}^{\dagger}\right)^{2}, \hat{\rho}_{b d}\right] \\
& +\kappa L[\hat{a}]\left(\hat{\rho}_{b d}\right)+\frac{\Gamma}{\Omega} Y L\left[\hat{a}+\hat{a}^{\dagger}\right] \hat{\rho}_{b d},
\end{aligned}
$$

plus terms of order $O\left(\omega_{0} \sqrt{\eta}\right)$. We defined $X=\Omega^{2} /\left(\Gamma^{2}+\Omega^{2}\right)$ and $Y=\frac{1}{4} \omega_{0} X g^{2}$. Since this equation is quadratic in $\hat{a}$, it can be solved by a Gaussian ansatz. The dynamics is then fully characterized by the evolution equation for the covariance matrix $\sigma$ of the state. The displacement vector decays quickly to zero and may be safely discarded, so we obtain $\partial_{t} \sigma=B \sigma+\sigma B^{T}-2 \kappa\left(\sigma-\sigma_{L}\right)$, where

$$
B=\left(\begin{array}{cc}
0 & \omega_{0} \\
4 Y-\omega_{0} & 0
\end{array}\right),
$$

and $\sigma_{L}=\frac{1}{2}\{\mathbb{I}+\operatorname{Diag}[0,4 Y \Gamma /(\Omega \kappa)]\}$. This linear equation may be solved exactly by diagonalization. Upon evaluating the lowest eigenvalue, one may estimate the typical time needed to reach the steady-state, $T \sim\left(g_{c} / 2 \kappa\right)(g-$ $\left.g_{c}\right)^{-1}\left(1+\omega_{0}^{2} / \kappa^{2}\right)^{-1}$. This value diverges near the transition, indicating a critical slowing down. The steady state is 
a squeezeed (undisplaced) thermal state, with covariance matrix given by

$$
\sigma=\left[\begin{array}{cc}
\frac{1}{2} & 0 \\
0 & \frac{2-g^{2} X(1-\delta)}{4}
\end{array}\right]+\frac{g^{2}(1+\delta)}{4\left(g_{c}^{2}-g^{2}\right)}\left[\begin{array}{cc}
1 & \frac{\kappa}{\omega_{0}} \\
\frac{\kappa}{\omega_{0}} & \left(\frac{\kappa}{\omega_{0}}\right)^{2}
\end{array}\right],
$$

with $g_{c}^{2}=\left(1+\Gamma^{2} / \Omega^{2}\right)\left(1+\kappa^{2} / \omega^{2}\right)$ and $\delta=\left(\omega_{0} \Gamma / \Omega \kappa\right)$. In this dissipative setting, the system still experiences a phase transition for $g \rightarrow g_{c}$. Both the squeezing and thermal energies of the steady-state diverge near the critical point. Since this state is Gaussian and its first-moment vector is zero, the QFI may be evaluated as (dots denote derivative with respect to the parameter $A$ under consideration)

$$
\mathcal{I}_{A}=\frac{8}{16 d^{4}-1}\left\{d^{4} \operatorname{Tr}\left[\left(\sigma^{-1} \dot{\sigma}\right)^{2}\right]-\frac{1}{4} \operatorname{Tr}\left[(\dot{\sigma} K)^{2}\right]\right\},
$$

with $d=\sqrt{\operatorname{Det} \sigma}$ and $K$ is the symplectic matrix [31]. The leading terms of the QFIs for the estimation of frequencies are given by

$$
\begin{aligned}
\mathcal{I}_{\omega_{0}}^{\text {diss }} & \sim \frac{2 \Omega}{\Omega \kappa+\omega_{0} \Gamma}\left(\frac{\kappa^{2}-\omega_{0}^{2}}{\kappa^{2}+\omega_{0}^{2}}\right)^{2}\langle\hat{N}\rangle T, \\
\mathcal{I}_{\Omega}^{\text {diss }} & \sim\left(\frac{\Gamma^{2}-\Omega^{2}}{\Gamma^{2}+\Omega^{2}}\right)^{2} \frac{\kappa^{2}}{2 \Omega^{2}}\left(1+\frac{\omega_{0}^{2}}{\kappa^{2}}\right)^{2} T^{2} .
\end{aligned}
$$

Equation (8) shows that for the estimation of $\omega_{0}$, the presence of dissipation restores the shot-noise scaling, similar to what happens in a lossy interferometric protocols. In the case in which the parameter to be estimated is the spin frequency $\Omega$, the presence of dissipation replaces the quartic time scaling obtained in the Hamiltonian case (6) by a quadratic one. However, the QFI of a Ramsey protocol in the presence of spin decay at rate $\Gamma$ is given by $\mathcal{I}_{\Omega}=T / \Gamma$, and so it is linear in time. This result shows that the timescaling enhancement of our critical protocol against the benchmark persists in the dissipative case for spinfrequency estimation.

Discussion.-Let us now comment on the nature, the limitations, and the potential experimental implementations of the considered protocols. First of all, we emphasize that our protocol exploits the diverging susceptibility near the transition, but it does not require to actually cross the critical point, contrary to what is used in transition-edge sensors [1]. The adiabatic evolution of the ground state is equivalent to applying a (time-dependent) squeezing Hamiltonian to the system. This procedure can be fit in the framework of quantum control, where it has been shown [32] that a quartic time scaling can be reached for the estimation of the frequency of rotation of a magnetic field.

Concerning the estimation of the spin frequency $\Omega$, our critical protocol achieves time-scaling enhancement compared to the Ramsey protocol. However, since the prefactor in Eqs. (6) and (8) is small, the critical protocol could outperform Ramsey schemes only for long protocol duration, i.e., when operating in close proximity of the critical point. In this region, the quartic and higher-order terms in the Schrieffer-Wolff expansion of the Hamiltonian, which have been neglected in order to obtain exact results, become relevant (Ref. [21] and the Supplemental Material [26]). A detailed study of the behavior of the QFI in this region requires numerical methods and goes beyond the reach of this work.

The proposed protocols could be implemented using analog quantum simulation techniques, which have already been experimentally applied for the quantum Rabi model in extreme regimes of parameters $[33,34]$ using different quantum technologies, such as cold atoms [35], superconducting circuits [36], and trapped ions [37]. Finitecomponent driven-dissipative phase transitions can be implemented with bath-engineering techniques [29]. Notice that these methods allow one to continuously tune the effective physical parameters, making it possible to reach and to implement the required adiabatic passage. Furthermore, it has been recently shown [23] that finitecomponent phase transitions can be observed also with weakly anharmonic quantum resonators, so our results could be extended to include a nonlinear quantum resonator implemented with circuit-QED devices [38] and electromechanical systems [39].

Conclusions and outlook.-Our results show that, in spite of the critical slowing down, critical quantum-optical systems represent a compelling tool for quantum metrology. In addition, we have demonstrated the metrological potential of finite-component quantum phase transitions, a result that has both practical and fundamental consequences. First, finite-component criticalities make it possible to substantially reduce the system size and complexity at the cost of accessing an unusual regime of parameters. Furthermore, we have shown that the adiabatic protocol can be optimized to reduce the protocol duration. This result reveals the potential of quantum-control schemes to reduce the time required to perform the adiabatic sweep in critical quantum metrology. Notice that in a finite-component system, quantum-control techniques could be applied without implementing complex nonlocal operations, as it is the case for many-body critical systems. Our study paves the way to the application of other criticalities appearing in quantum-optical models $[23,40-42]$ in quantum metrology. Finally, by focusing on the time scaling and on a finite-component system, our analysis challenges the standard framework in which the fundamental resources needed to achieve metrological quantum advantage are assessed [43-46].

We thank Philipp Schneeweiss for useful discussions. S. F. acknowledges support from the European Research Council (ERC-2016-STG-714870). 
*Corresponding author. simone.felicetti@cnr.it

[1] K. Irwin and G. Hilton, in Cryogenic Particle Detection, Topics in Applied Physics, edited by C. Enss (Springer, Berlin Heidelberg, 2005), pp. 63-150.

[2] R. Demkowicz-Dobrzański, M. Jarzyna, and J. Kolodyński, in Progress in Optics, edited by E. Wolf (Elsevier, 2015), Vol. 60, pp. 345-435, https://www.sciencedirect.com/ science/article/pii/S0079663815000049?via\%3Dihub.

[3] M. Tsang, Phys. Rev. A 88, 021801(R) (2013).

[4] K. Macieszczak, M. Guţă, I. Lesanovsky, and J. P. Garrahan, Phys. Rev. A 93, 022103 (2016).

[5] P. Zanardi, M. G. A. Paris, and L. C. Venuti, Phys. Rev. A 78, 042105 (2008).

[6] M. Bina, I. Amelio, and M. G. A. Paris, Phys. Rev. E 93, 052118 (2016).

[7] P. A. Ivanov, Phys. Scr. 95, 025103 (2020).

[8] S. Fernández-Lorenzo and D. Porras, Phys. Rev. A 96, 013817 (2017).

[9] M. M. Rams, P. Sierant, O. Dutta, P. Horodecki, and J. Zakrzewski, Phys. Rev. X 8, 021022 (2018).

[10] T. L. Heugel, M. Biondi, O. Zilberberg, and R. Chitra, Phys. Rev. Lett. 123, 173601 (2019).

[11] P. A. Ivanov and D. Porras, Phys. Rev. A 88, 023803 (2013).

[12] C. Invernizzi, M. Korbman, L. C. Venuti, and M. G. A. Paris, Phys. Rev. A 78, 042106 (2008).

[13] S. Sachdev, Quantum Phase Transitions (Cambridge University Press, Cambridge, 2011), https://doi.org/10 .1017/CBO9780511973765.

[14] K. Macieszczak, M. Guţă, I. Lesanovsky, and J. P. Garrahan, Phys. Rev. Lett. 116, 240404 (2016).

[15] F. Minganti, A. Biella, N. Bartolo, and C. Ciuti, Phys. Rev. A 98, 042118 (2018).

[16] P. Kirton, M. M. Roses, J. Keeling, and E. G. Dalla Torre, Adv. Quantum Technol. 2, 1800043 (2019).

[17] Z. Zhiqiang, C. H. Lee, R. Kumar, K. Arnold, S. J. Masson, A. Parkins, and M. Barrett, Optica 4, 424 (2017).

[18] K. Baumann, C. Guerlin, F. Brennecke, and T. Esslinger, Nature (London) 464, 1301 (2010).

[19] L. Bakemeier, A. Alvermann, and H. Fehske, Phys. Rev. A 85, 043821 (2012).

[20] S. Ashhab, Phys. Rev. A 87, 013826 (2013).

[21] M.-J. Hwang, R. Puebla, and M. B. Plenio, Phys. Rev. Lett. 115, 180404 (2015).

[22] J. Peng, E. Rico, J. Zhong, E. Solano, and I. L. Egusquiza, Phys. Rev. A 100, 063820 (2019).

[23] S. Felicetti and A. Le Boité, Phys. Rev. Lett. 124, 040404 (2020).

[24] S. F. Huelga, C. Macchiavello, T. Pellizzari, A. K. Ekert, M. B. Plenio, and J. I. Cirac, Phys. Rev. Lett. 79, 3865 (1997).

[25] V. Giovannetti, S. Lloyd, and L. Maccone, Science 306, 1330 (2004).
[26] See Supplemental Material at http://link.aps.org/supplemental/ 10.1103/PhysRevLett.124.120504 for an analysis of the metrological potential of the superradiant phase, an analysis of the precision achievable with homodyne measurements, technical details related to the adiabatic process and the dissipative case, and a detailed discussion of the limits of validity of our model.

[27] M. A. C. Rossi, M. Bina, M. G. A. Paris, M. G. Genoni, G. Adesso, and T. Tufarelli, Quantum Sci. Technol. 2, 01LT01 (2017).

[28] T.-L. Wang, L.-N. Wu, W. Yang, G.-R. Jin, N. Lambert, and F. Nori, New J. Phys. 16, 063039 (2014).

[29] R. Puebla, M.-J. Hwang, J. Casanova, and M. B. Plenio, Phys. Rev. Lett. 118, 073001 (2017).

[30] M.-J. Hwang, P. Rabl, and M. B. Plenio, Phys. Rev. A 97, 013825 (2018).

[31] A. Monras, preprint at arXiv:1303.3682.

[32] S. Pang and A. N. Jordan, Nat. Commun. 8, 14695 (2017).

[33] P. Forn-Díaz, L. Lamata, E. Rico, J. Kono, and E. Solano, Rev. Mod. Phys. 91, 025005 (2019).

[34] A. F. Kockum, A. Miranowicz, S. De Liberato, S. Savasta, and F. Nori, Nat. Rev. Phys. 1, 19 (2019).

[35] A. Dareau, Y. Meng, P. Schneeweiss, and A. Rauschenbeutel, Phys. Rev. Lett. 121, 253603 (2018).

[36] J. Braumüller, M. Marthaler, A. Schneider, A. Stehli, H. Rotzinger, M. Weides, and A. V. Ustinov, Nat. Commun. 8, 779 (2017).

[37] D. Lv, S. An, Z. Liu, J.-N. Zhang, J. S. Pedernales, L. Lamata, E. Solano, and K. Kim, Phys. Rev. X 8, 021027 (2018).

[38] D. Marković, S. Jezouin, Q. Ficheux, S. Fedortchenko, S. Felicetti, T. Coudreau, P. Milman, Z. Leghtas, and B. Huard, Phys. Rev. Lett. 121, 040505 (2018).

[39] G. A. Peterson, S. Kotler, F. Lecocq, K. Cicak, X. Y. Jin, R. W. Simmonds, J. Aumentado, and J. D. Teufel, Phys. Rev. Lett. 123, 247701 (2019).

[40] N. Bartolo, F. Minganti, W. Casteels, and C. Ciuti, Phys. Rev. A 94, 033841 (2016).

[41] W. Casteels, R. Fazio, and C. Ciuti, Phys. Rev. A 95, 012128 (2017).

[42] L. Garbe, I. L. Egusquiza, E. Solano, C. Ciuti, T. Coudreau, P. Milman, and S. Felicetti, Phys. Rev. A 95, 053854 (2017).

[43] P. Hyllus, O. Gühne, and A. Smerzi, Phys. Rev. A 82, 012337 (2010).

[44] B. Yadin, F. C. Binder, J. Thompson, V. Narasimhachar, M. Gu, and M. S. Kim, Phys. Rev. X 8, 041038 (2018).

[45] H. Kwon, K. C. Tan, T. Volkoff, and H. Jeong, Phys. Rev. Lett. 122, 040503 (2019).

[46] L. Garbe, S. Felicetti, P. Milman, T. Coudreau, and A. Keller, Phys. Rev. A 99, 043815 (2019). 